\title{
Densidade de esporos de fungos micorrízicos arbusculares em uma cronossequência de florestas secundárias na Amazônia oriental
} Density of spores of arbuscular mycorrhizal fungi in a chronosequence of secondary forests in eastern Amazonia

\author{
Priscila Sanjuan de Medeiros', Leandro Valle Ferreirall \\ 'Universidade do Estado do Pará. Belém, Pará, Brasil \\ "Museu Paraense Emílio Goeldi/MCTIC. Belém, Pará, Brasil
}

\begin{abstract}
Resumo: Nos solos pobres das regiões tropicais, a formação de associações micorrízicas é de extrema importância para a sobrevivência e o crescimento das plantas. O objetivo deste estudo foi avaliar a densidade de fungos micorrízicos arbusculares (FMA) em uma cronossequência de florestas secundárias e primárias, entre os períodos seco e chuvoso, na Floresta Nacional de Caxiuanã, Pará. Foram selecionadas 40 áreas, sendo três de floresta primária e 37 de floresta secundária, com diferentes idades. A cada campanha (três no período menos chuvoso e três no chuvoso), foram coletadas três amostras de solo por área. Não houve relação entre a densidade de FMA e a idade da floresta. Encontrou-se maior número de esporos na primeira camada do solo e na estação seca. Observou-se que o número de esporos é maior com o aumento da umidade, da quantidade de raízes finas e da concentração de N, K e argila total no solo. Embora os fungos micorrízicos venham sendo usados amplamente como bioindicadores, os resultados demonstram que o uso da densidade de FMA como indicador em florestas secundárias de terra firme, na região amostrada, limita-se à separação entre floresta secundária e primária, não sendo possível separar as diferentes etapas do processo sucessional.
\end{abstract}

Palavras-chave: Amazônia. Sucessão. Sazonalidade. Solo. Fungos micorrízicos arbusculares.

Abstract: In the tropics where most soil is infertile, the formation of mycorrhizal associations is of extreme importance for plant survival and growth. The objective of this study was to evaluate the arbuscular mycorrhizal fungi (AMF) density in a chronosequence of secondary and primary forests, between the dry and rainy seasons in the Caxiuanã National Forest, Pará, Brazil. Forty areas were selected, three areas of primary forest and 37 areas of secondary forests of different ages. On every excursion (three in the dry season and three in the rainy season), three soil samples were collected in each area. There was no relationship between AMF density and age of the forest. The number of spores was seen to increase with increasing moisture, with the amount of fine roots and the concentration of $\mathrm{N}, \mathrm{K}$ and the total clay in the soil. Although mycorrhizal fungi have been widely used as bioindicators, the data demonstrate that the use of AMF density as an indicator in secondary upland forest, is limited to separating primary forests from secondary forests, and it is not possible to separate the different stages of the successional process.

Keywords: Amazon. Succession. Seasonality. Soil. Arbuscular mycorrhizal fungi.

MEDEIROS, P. S. \& L. V. FERREIRA, 2018. Densidade de esporos de fungos micorrízicos arbusculares em uma cronossequência de florestas secundárias na Amazônia Oriental. Boletim do Museu Paraense Emílio Goeldi. Ciências Naturais 13(2): 247-259.

Autora para correspondência: Priscila Sanjuan de Medeiros. Centro de Ciências Naturais e Tecnologia. Tv. Enéas Pinheiro, 2626 - Marco. Belém, PA, Brasil. CEP 66095-105 (priscilasanjuanbio@yahoo.com.br).

Recebido em 07/08/2017

Aprovado em 22/05/2018

Responsabilidade editorial: Fernando da Silva Carvalho Filho

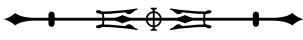




\section{INTRODUÇÃO}

Os fungos micorrízicos arbusculares (FMA) são organismos biotróficos obrigatórios que se associam com raízes, formando uma relação simbiótica mutualista, denominada de micorriza arbuscular (Souza et al., 2010). Colonizam cerca de $90 \%$ das espécies de plantas vasculares, ocorrendo em quase todos os gêneros de gimnospermas e de angiospermas (Berbara et al., 2006), além de alguns representantes de briófitas e de pteridófitas (F. Moreira \& J. Siqueira, 2006).

$\mathrm{Na}$ associação micorrízica arbuscular, a planta cede a energia necessária (fotossintatos) para o crescimento e a reprodução dos fungos, que, em troca, absorvem nutrientes e os disponibilizam para células do córtex das raízes (Souza et al., 2008). Além disso, os FMA promovem o aumento da superfície de absorção das raízes, atuando principalmente na assimilação de nutrientes que possuem baixa mobilidade, resultando no aumento da tolerância da planta a estresses abióticos e bióticos (Augé, 2001; L. Maia \& A. Yano-Melo, 2005; Smith \& Read, 2008).

Em algumas espécies de plantas, a dependência micorrízica é acentuada, sendo que, na ausência de simbiose, as plantas não conseguem absorver os nutrientes necessários para o seu desenvolvimento (Souza et al., 2010), podendo suscitar mudanças na estrutura da paisagem (Miller \& Kling, 2000). Além dos benefícios proporcionados às plantas, os FMA trazem melhorias para a estruturação dos solos (Bonfim, 2011), uma vez que o micélio externo é responsável pela exsudação de glicoproteínas hidrofóbicas, chamadas de glomalinas, as quais atuam como agentes cimentantes das partículas do solo, agindo diretamente na formação de agregados estáveis (Bai et al., 2009; Purin \& Rillig, 2007; Rillig et al., 2010).

A intensificação do uso do solo na Amazônia tem aumentado gradativamente as regiões cobertas por florestas secundárias (Kennard, 2002; Lanly, 1982). Alterações como estas promovem modificações nos atributos físicos, químicos e biológicos do solo, acarretando prejuízos para a sustentabilidade dos ecossistemas, bem como no que se refere ao poder de resiliência da floresta (Cordeiro et al., 2005; Oehl et al., 2004).

A primeira etapa do processo sucessional em florestas tropicais é caracterizada pela presença de espécies vegetais de crescimento rápido (Chazdon, 2012), as quais geralmente crescem em áreas pobres em nutrientes. Como o processo de recuperação do solo é lento e gradativo, é de se esperar que essas espécies apresentem estratégias específicas para superar as deficiências do solo (Janos, 1996). Na floresta amazônica, a densidade de esporos de FMA no solo é maior durante o início do processo de sucessão vegetal (Freitas, 2005; Stürmer \& Siqueira, 2008). Isso seria justificável em razão de, nessa fase, a associação entre o fungo e a planta tornar-se mais intensa, devido à deficiência de algumas substâncias essenciais ao crescimento da planta (Zangaro et al., 2009).

Embora os fungos micorrízicos arbusculares sejam considerados essenciais para o desenvolvimento da vegetação na floresta tropical úmida (Janos, 1980), especialmente em florestas secundárias (Zangaro et al., 2009), ainda há poucas informações sobre as variações da comunidade de FMA nestes ecossistemas. Dessa forma, o objetivo deste estudo foi avaliar a densidade de FMA em uma cronossequência de florestas secundárias, com idades de 1 a 40 anos, e em florestas primárias, a fim de entender e de estabelecer padrões desses organismos no processo de sucessão em floresta de terra firme, tomando como base a Floresta Nacional de Caxiuanã, no estado do Pará.

\section{MATERIAL E MÉTODOS}

\section{ÁREA DE ESTUDO}

O estudo foi realizado na Estação Cientíica Ferreira Penna, localizada na Floresta Nacional de Caxiuanã (10 13' 86" S; $48^{\circ}$ 17' 41,18” W), no município de Melgaço, estado do Pará (Lisboa, 1997). O clima da região, de acordo com a classificação de Köppen, é do tipo tropical, quente e úmido, e do subtipo climático 'Am'. A temperatura média do ar é de $26,7^{\circ} \mathrm{C}$, oscilando entre o mínimo de $22^{\circ} \mathrm{C}$ e 
o máximo de $32{ }^{\circ} \mathrm{C}$ (A. Costa et al., 2012). A região está inserida em uma área de planície, com relevo apresentando altitudes inferiores a $50 \mathrm{~m}$, caracterizado por interflúvios extensos e tabulares, com maior distribuição de solo classificado como tipo latossolo amarelo (M. L. Costa et al., 2002). A precipitação anual não ultrapassa 3.000 mm. Climatologicamente, o período chuvoso está compreendido entre os meses de janeiro a junho, e o período mais seco, entre julho a dezembro (Moraes et al., 2009).

\section{COLETA DE DADOS}

Para o estudo em floresta secundária, adotou-se a metodologia de cronossequência, a qual estabelece uma substituição do tempo pelo espaço (Chazdon, 2012). Nesse método, assume-se que áreas espacialmente diferentes e com variados tempos de regeneração sob condições bióticas e abióticas similares e com usos equivalentes - constituem uma representação temporal do processo de sucessão (Wildi, 2002). Foram selecionadas 40 áreas, com tamanho médio de 0,75 hectare, sendo três de floresta primária de terra firme e 37 de floresta secundária, com idades variando de 1 a 40 anos. Foram realizadas seis campanhas a cada dois meses, três no período chuvoso e três no período menos chuvoso, entre julho de 2013 e maio de 2014. Em cada uma, todas as variáveis foram medidas. A amostragem de solo para a quantificação do número de esporos de fungos micorrízicos arbusculares foi realizada utilizando-se um tubo indeformável, de 10 $\mathrm{cm}$ de altura, em duas camadas do solo, de 0-10 cm e de 10-20 cm. Em cada local, foram coletadas três amostras por camada a cada campanha.

Os esporos dos FMA foram extraídos de $50 \mathrm{~g}$ de solo pela metodologia de peneiramento úmido, em malha de 0,710 mm e 0,053 mm (Gerdemann \& Nicolson, 1963), seguida pelo processo de centrifugação em água (3.000 rpm por 3 minutos) e em sacarose 50\% (2.000 rpm por dois minutos) (Jenkins, 1964). O sobrenadante passou pelo peneiramento úmido, em malha de 0,053 $\mathrm{mm}$. A contagem dos esporos foi realizada por meio de uma placa canaletada, com auxílio de microscópio estereoscópio (40x).

Para avaliação da umidade gravimétrica, em cada parcela $(20 \times 20 \mathrm{~m})$, foram retiradas três amostras de solo utilizando-se tubo coletor com $6,5 \mathrm{~cm}$ de diâmetro por 10 $\mathrm{cm}$ de profundidade. Uma pesagem da amostra de solo foi realizada assim que foi retirada e outra foi feita após secagem em estufa, a $105^{\circ} \mathrm{C}$ por $48 \mathrm{~h}$ (EMBRAPA, 1997). A umidade gravimétrica foi expressa em porcentagem e obtida conforme a fórmula: $\cup g=($ (peso úmido - peso seco)/peso) x 100 (EMBRAPA, 1997).

Foi retirada uma amostra composta do solo para avaliação de atributos químicos e físicos de cada área, de acordo com os procedimentos recomendados pela EMBRAPA (1997). As amostras foram enviadas para o laboratório de solos da EMBRAPA Amazônia Oriental, onde foram analisados a quantidade de matéria orgânica no solo, a fertilidade ( $\mathrm{K}, \mathrm{P}, \mathrm{Na}, \mathrm{Ca}, \mathrm{Mg}, \mathrm{Al})$, o N, o pH e a granulometria (areias grossa e fina, silte e argila total).

Para quantificar a luminosidade em cada parcela, calculou-se a abertura de dossel. Esta estimativa foi feita a partir de imagens captadas com câmera fotográfica digital e do uso do programa Imagej, no qual a proporção de pixels da área coberta é calculada após conversão da imagem original para preto e branco, na qual a vegetação se ilustra em preto (cobertura de dossel) e o céu (abertura de dossel) em branco. Para cada parcela, obteve-se a média da abertura do dossel a partir de cinco imagens.

A estimativa da biomassa das raízes finas (diâmetro $<2 \mathrm{~mm}$ ) foi obtida por meio do peso das raízes por volume de solo de cada amostra (Barbosa \& Santos, 2009). Assim como nos solos, por coleta, foram retiradas três amostras em cada parcela $(20 \times 20 \mathrm{~m})$, as quais também foram coletadas com auxílio de um tubo de 6,5 cm de diâmetro por $10 \mathrm{~cm}$ de profundidade. Para a separação das raízes finas, as amostras de solo foram peneiradas, em malha de $2 \mathrm{~mm}$, em água corrente. Depois de separadas, as raízes foram colocadas em sacos de papel e levadas a estufa a $60{ }^{\circ} \mathrm{C}$ por 48 horas, para 
obtenção do peso constante, a fim de se efetivar posterior pesagem em balança analítica (Barbosa \& Santos, 2009).

Com o objetivo de se obter a densidade e a riqueza de árvores e de arbustos nas áreas estudadas, bem como de relacionar estas variáveis com a densidade de FMA, realizou-se levantamento florístico dos indivíduos com diâmetro a altura do peito (DAP) superior a $1 \mathrm{~cm}$. As espécies foram identificadas por um parataxonomista do Museu Paraense Emílio Goeldi, de acordo com o sistema de classificação de The Angiosperm Phylogeny Group (APG, 2009).

\section{ANÁLISE DOS DADOS}

A fim de verificar qual o melhor método para a análise das variáveis bióticas e abióticas das florestas secundárias, utilizou-se o fator idade como uma variável contínua e discreta, sendo que a categorização seguiu o sugerido por Chazdon (2008). Nessa classificação, as florestas secundárias são separadas de acordo com a idade em quatro etapas: etapa 1 - parcelas entre um e dez anos de regeneração pós-abandono; etapa 2 - entre 11 e 25 anos; etapa 3 - entre 26 e 45 anos; e etapa 4 - referente às áreas-controles.

A relação entre a densidade de esporos de fungos micorrízicos arbusculares (variáveis dependentes) e a idade das florestas secundárias (variável independente) foi testada por meio de um modelo de regressão linear simples (Gotelli \& Ellison, 2004).

As diferenças da densidade de fungos micorrízicos arbusculares entre as quatro etapas sucessionais foram testadas por meio de um modelo de análise de variância simples (ANOVA) de um fator. Para diminuir o efeito do desbalanceamento do tamanho das amostras, optou-se pela metodologia da soma de quadrados tipo III (Coimbra et al., 2006). Quando o valor obtido na ANOVA foi significativo, a posteriori o teste de tuckey foi aplicado, a fim de identificar quais diferenças são significativas par a par (Zar, 2009). Quando os pressupostos da análise não foram atingidos (amostras independentes, homogeneidade das variâncias entre os grupos, resíduos com distribuição normal), o teste equivalente não paramétrico Kruskal-Wallis e, a posteriori, o teste de Dunnett foram usados (Zar, 2009).

O teste $\mathrm{t}$ de Student - ou equivalente não paramétrico teste $t$ com variâncias separadas - foi usado para verificar diferenças da variável dependente (densidade) entre os períodos chuvoso e menos chuvoso (tratamento) (Zar, 2009). Para se determinar o efeito da idade da floresta secundária sobre a densidade de FMA, excluindo-se o do período de coleta (chuvoso e menos chuvoso) e da profundida do solo das quais as amostras foram coletadas (0-10 ou 10-20 cm), utilizou-se um modelo de Análise de Covariância (ANCOVA) (Zar, 2009).

Os dados foram analisados por meio de análise de variância fatorial, a fim de se testar a hipótese de que a etapa do processo sucessional afeta a densidade de FMA, bem como verificar se este efeito depende do período de coleta (chuvoso e menos chuvoso) e da profundidade do solo de onde as amostras foram coletadas (0-10 ou 10-20 cm) (Zar, 2009).

Utilizou-se análise de correlação a fim de se testar o efeito das variáveis microclimáticas (umidade de solo, luminosidade), bem como de variáveis físicas do solo (areias grossa e fina, silte e argila), químicas ( $\mathrm{N}, \mathrm{pH}, \mathrm{MO}$, $\mathrm{P}, \mathrm{K}, \mathrm{Na}, \mathrm{Ca}, \mathrm{Mg}, \mathrm{Al}$ ), além das variáveis da vegetação (riqueza, densidade e idade) na densidade de FMA (variáveis dependentes) (Zar, 2009).

Os pacotes estatísticos utilizados foram: $R \circledast(\mathrm{v}$. 3.2.0), PRIMER ${ }^{\circledR}$ ( v. 6 Beta), Statistica ${ }^{\circledR}$ (v. 7.2.4.1) e PC-ORD ${ }^{\circledR}$ (v. 5.15). Para todas as análises, foram testados os pressupostos, sendo o nível de significância fixado em 0,05.

\section{RESULTADOS}

Não houve relação significativa entre a densidade de esporos de fungos micorrízicos arbusculares (FMA) e a idade da floresta secundária $\left(r^{2}=0,001 ; p=0,5\right)$ (Figura 1), sendo o mesmo padrão observado ao se realizar a

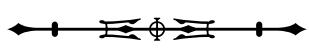




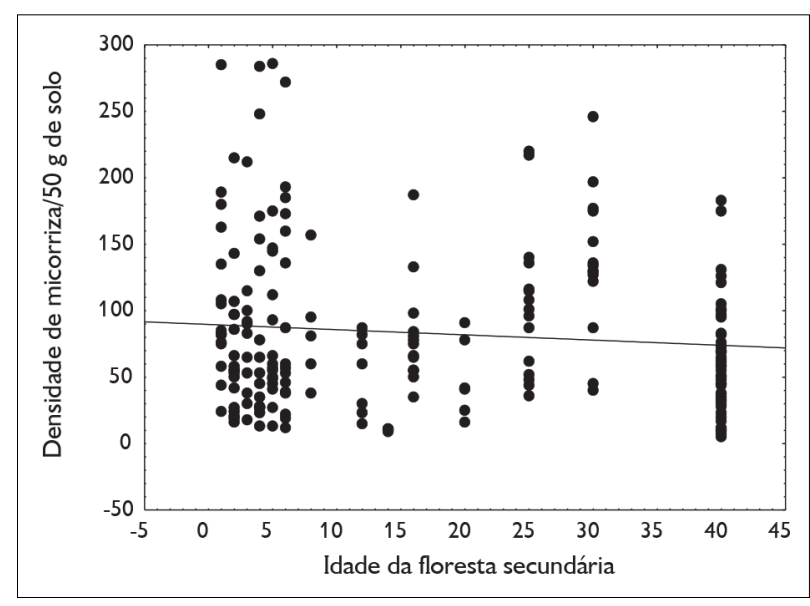

Figura 1. Densidade de esporos de FMA em relação à idade da floresta secundária na FLONA de Caxiuanã, Pará.

análise separadamente para as amostras coletadas em profundidade de $0-10 \mathrm{~cm}\left(r^{2}=0,001 ; p=0,5142\right)$ e 10 $20 \mathrm{~cm}\left(r^{2}=0,002 ; p=0,509\right)$.

Ao se isolar o efeito do período da coleta (chuvoso e menos chuvoso) ( $F=0,92 ; p=0,33$; Figura $2 A)$ e a profundidade na qual as amostras foram coletadas $(F=$ 2,3; $p=0,13$; Figura 2B), não houve relação significativa entre a densidade de esporos de FMA e a idade da floresta secundária.

A densidade de esporos de FMA foi significativamente menor na etapa 4 (florestas primárias), em comparação com as etapas 1 a 3, que não diferem entre si $(H=29.88$; p < 0,001; Figura 3). $\bigcirc$ mesmo padrão foi observado ao se fazer a análise separando as duas profundidades de coleta $(0-10 \mathrm{~cm}: \mathrm{H}=15,89 ; \mathrm{p}=0,001 / 10-20 \mathrm{~cm}: \mathrm{H}=$ 17,57; $p=0,0005)$.

A densidade de esporos de FMA foi consideravelmente maior na primeira camada do solo (0-10 cm) em comparação com a segunda camada (10$20 \mathrm{~cm})(t=5,82 ; p<0,0001)$ (Figura 4).

A densidade de esporos de FMA é substancialmente maior na estação menos chuvosa em comparação com a chuvosa nas duas profundidades analisadas (Figura $5 A-0-10 \mathrm{~cm}: t=2,08 ; p=0,02 /$ Figura $5 B-10-20 \mathrm{~cm}$ : $t=2,36 ; p=0,020$ ).

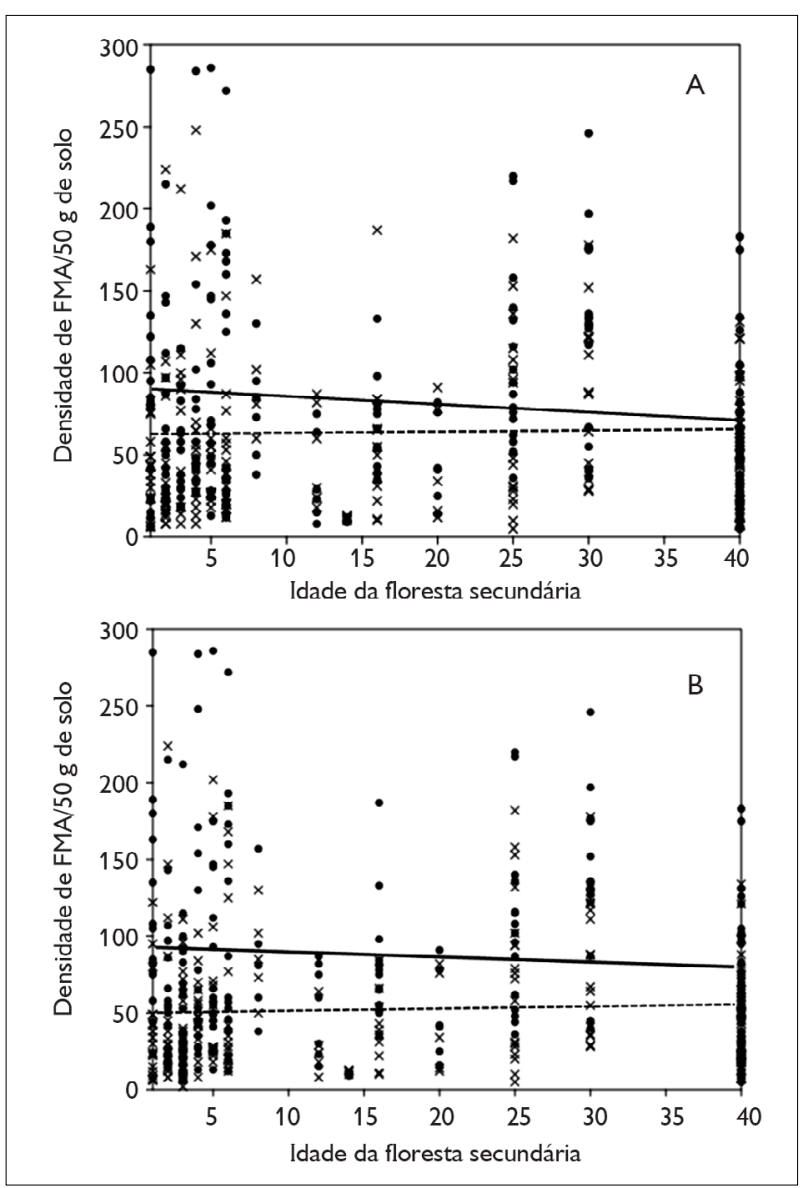

Figura 2. Relação entre a densidade de FMA e a idade da floresta secundária nos períodos $\operatorname{seco}(\bullet)$ e chuvoso $(x)(A)$ e na profundidade de 0-10 (•) e 10-20 cm (x) (B).

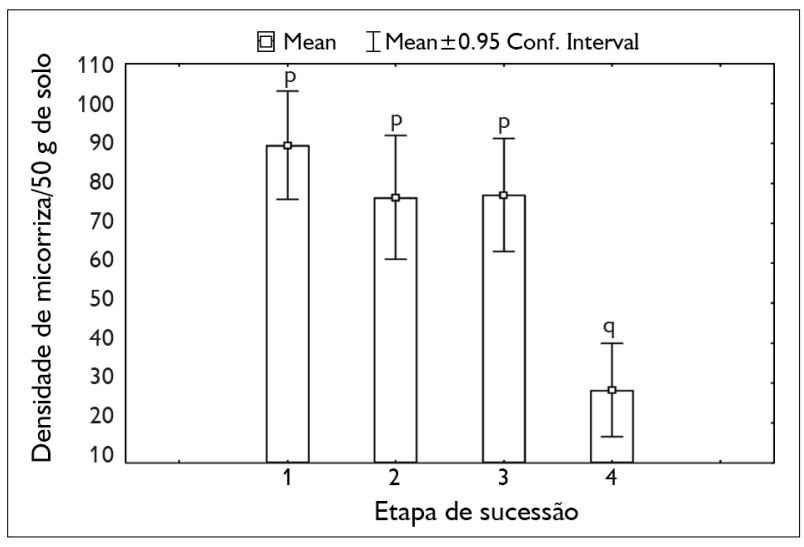

Figura 3. Densidade de esporos de FMA entre as quatro etapas do processo sucessional na FLONA de Caxiuanã, Pará. Letras distintas representam médias estatisticamente diferentes a 95\% de probabilidade. 


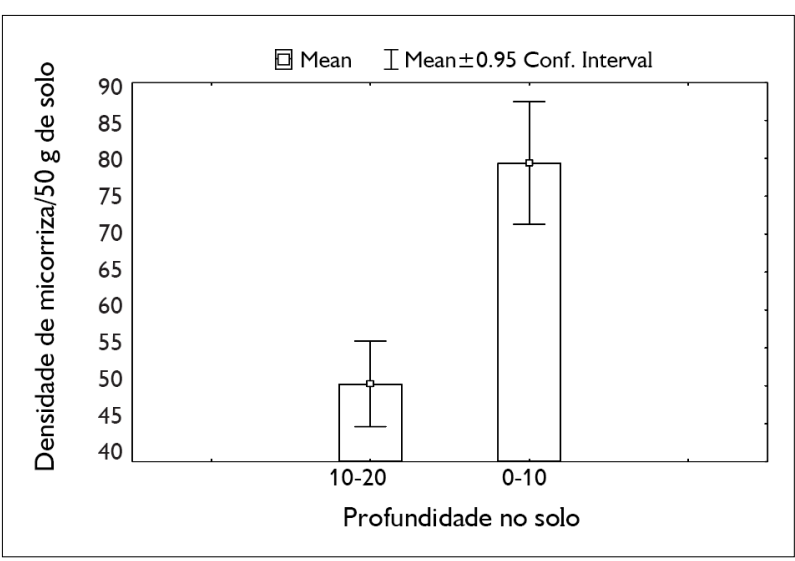

Figura 4. Média da densidade de esporos de FMA entre as duas diferentes camadas do solo amostradas.

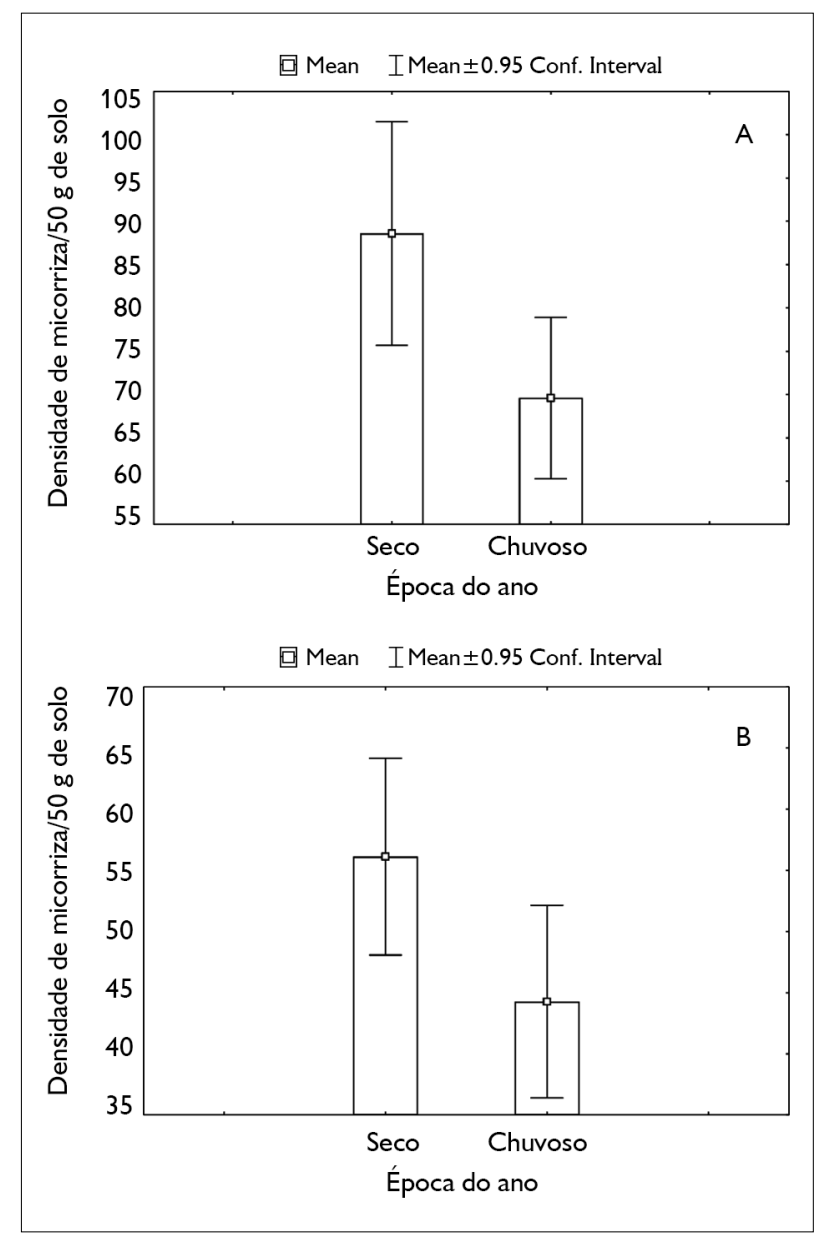

Figura 5. Média da densidade de esporos de FMA na profundidade de $0-10 \mathrm{~cm}(A)$ e de $10-20 \mathrm{~cm}(B)$ entre os períodos seco e chuvoso.
A interação entre fatores mostra que a profundidade do solo e o período de coleta não influenciam diferentemente a densidade de FMA nas diferentes etapas do processo sucessional (Tabela 1). Ou seja, independentemente da época de coleta e da profundidade na qual o solo é amostrado, há diferença na densidade de FMA nas quatro etapas do processo sucessional.

A densidade de esporos de FMA foi significativamente influenciada pela quantidade de raízes finas, pela umidade do solo, pela riqueza de espécies arbóreas, pelas concentrações de $\mathrm{K}$ e $\mathrm{N}$ e pela porcentagem de areia fina e de argila no solo. Correlação positiva foi observada entre o número de esporos e a umidade do solo $(r=$ $0,13 ; p<0,05)$, a quantidade de raízes finas $(r=0,20$; $p<0,05)$, as concentrações de $N(r=0,12 ; p<0,05)$, de $K(r=0,14 ; p<0,05)$ e de argila total $(r=0,10 ; p$ $<0,002)$, enquanto que a riqueza de espécies arbóreas $(r=-0,12 ; p<0,05)$ e a porcentagem de areia fina $(r=$ $-0,20 ; p<0,05)$ mostraram correlação negativa com o número de esporos.

\section{DISCUSSÃO}

A diminuição do número de esporos de fungos micorrízicos arbusculares (FMA) em relação ao progresso do processo sucessional é descrita por diversos autores em estudos referentes à Mata Atlântica (Aidar et al., 2004; Stürmer

Tabela 1. ANOVA fatorial considerando os efeitos da etapa do processo sucessional (1 a 4), o período de coleta (chuvoso e menos chuvoso), a profundidade em que o solo foi coletado $(0-10 \mathrm{~cm}$ e $10-20 \mathrm{~cm})$ e suas interações na densidade de fungos micorrízicos arbusculares.

\begin{tabular}{c|c|c}
\hline & \multicolumn{2}{|c}{ Densidade FMA } \\
\hline Efeito & $F$ & $P$ \\
\hline Etapa & 6.623 & 0.001 \\
\hline Período & 3.356 & 0.049 \\
\hline Profundidade & 11.979 & 0.001 \\
\hline Período $\times$ etapa & 0.782 & 0.504 \\
\hline Período $\times$ profundidade & 0.002 & 0.962 \\
\hline Etapa $\times$ profundidade & 1.324 & 0.265 \\
\hline Período $\times$ etapa $\times$ profundidade & 1.413 & 0.238 \\
\hline
\end{tabular}


et al., 2006; Zangaro et al., 2008; Bonfim et al., 2010) e à floresta amazônica (Freitas, 2005; Stürmer \& Siqueira, 2008). De acordo com Guadarrama \& Álvarez-Sánches (1999) e Miranda (2008), é esperada a diminuição da densidade de esporos de FMA, em razão do aumento da idade da floresta secundária, uma vez que a produção de esporos de FMA é um mecanismo de perpetuação das espécies, sendo estimulada quando a planta e o fungo são submetidos a estresses ambientais. No entanto, na área de estudo, a relação entre a densidade de FMA e a idade da floresta secundária não foi significativa, apesar de ter sido possível observar a diminuição do número de esporos com o aumento da idade da floresta.

Essa diferença entre os resultados encontrados na literatura e o observado nesse estudo pode estar relacionada a maneiras diversas de se analisar e se interpretar os dados. Todos os estudos citados avaliaram dados categóricos, ou seja, diferentes tratamentos (floresta secundária jovem, tardia e floresta primária), e não uma variável contínua (áreas de diferentes idades). Portanto, não há base para se afirmar que a densidade de FMA diminui com o aumento da idade da floresta secundária. O que observamos nos estudos citados é a mesma relação encontrada quando categorizamos o processo sucessional, uma vez que há diferentes densidades de FMA nas quatro etapas do processo sucessional. No entanto, só há diferença significativa entre a floresta primária (etapa 4) e as florestas secundárias (etapas 1 a 3).

Stürmer \& Siqueira (2008), avaliando a ocorrência e a diversidade de FMA sob diferentes sistemas de uso na Amazônia (floresta, capoeiras velha e nova, sistema agroflorestal, culturas e pastagens), verificaram que a densidade de esporos na floresta nativa foi significativamente menor, quando comparada à de outros sistemas de uso do solo, sendo os maiores números registrados em capoeiras novas e pastagem. Caproni et al. (2003), estudando um local em processo de restauração após mineração de bauxita, no estado do Pará, observaram que as áreas em recuperação produziram mais esporos de FMA do que as da floresta primária adjacente. A literatura também aponta maior número de esporos em áreas agrícolas quando comparadas à vegetação natural (Miranda et al., 2005; M. Moreira et al., 2006; Carrenho et al., 2010). Esse fato pode explicar o maior número de esporos encontrado na etapa 1, visto que estas áreas apresentam histórico de cultivo agrícola mais recente (Medeiros, 2016).

A maior densidade de esporos na etapa 1 também pode ser justificada pelo fato de ser encontrado nestas áreas maior número de indivíduos vegetais ainda não desenvolvidos completamente, o que aumenta a dependência dos FMA (Zangaro et al., 2002). Segundo Daniels-Hetrick \& Bloom (1986), uma maior esporulação por FMA é atribuída aos hospedeiros que apresentam grande capacidade de fornecer fotoassimilados ao fungo, ou seja, plantas de rápido crescimento. Nos estágios iniciais, a floresta secundária é caracterizada por maior incidência de radiação luminosa nos solo, provocando rápidos ciclos de maior e de menor umidade, o que pode induzir à esporulação (Zangaro \& Moreira, 2010).

O menor número de esporos na floresta primária, em relação às secundárias, confirma a maior atuação dos FMA em solos em recuperação que ainda respondem a processos de perturbação (Piotrowski et al., 2008). Essa diferença pode ser justificada pelas características das plantas, que também mudam ao longo do processo sucessional, principalmente devido às condições às quais precisam se adaptar. As plantas que ocorrem nos primeiros anos da sucessão apresentam alta demanda por luz, requerem ambientes abertos e apresentam taxa de crescimento rápido, enquanto que as espécies em sucessões mais antigas e na floresta primária são tolerantes à sombra e têm crescimento lento (Khurana \& Singh, 2006). Sendo assim, é de se esperar que áreas com composição vegetal similares apresentem uma comunidade de FMA com características também similares. $\mathrm{Na}$ área de estudo, observou-se que não há diferença significativa na densidade de FMA entre 
as etapas 2 e 3 , fato que pode ser justificado pela composição de espécies arbustivas e arbóreas, similares nas etapas 2 e 3 (Medeiros, 2016).

Outros autores atribuem a menor densidade de FMA na floresta primária à maior estabilidade do ecossistema, com menor competição por nichos, permitindo a multiplicação dos FMA, mesmo com taxa de esporulação baixa (Munyanziza et al., 1997; Caproni et al., 2003; Oehl et al., 2003).

fato de diversos estudos citarem baixa densidade de FMA em floresta primária não indica baixa colonização, mas sim a possibilidade de os esporos serem relativamente pouco importantes como propágulos neste ecossistema (Muthukumar et al., 2003). Em solos não perturbados ou em com o mínimo de perturbação, a colonização de novas raízes se dá principalmente através da rede de hifas (Jasper et al., 1989).

Estudos que avaliam a estrutura das comunidades de FMA são geralmente restritos às camadas mais superficiais do solo (Oehl et al., 2005). Eles demonstram que aumento da profundidade acarreta diminuição no número de esporos de FMA, assim como produz o decréscimo na percentagem de raízes colonizadas (Muthukumar et al., 2003; Cardoso et al., 2003; Yang et al., 2010; Bonfim, 2011; Rillig \& Field, 2003). Tal fato pode estar associado à predominância de raízes finas nas camadas mais superficiais, já que os esporos são altamente dependentes das raízes, principalmente das mais finas, para iniciarem a simbiose (Cardoso et al., 2003; Zangaro et al., 2008). Dessa forma, aparentemente a densidade de esporos acompanha a densidade de raízes finas ao longo do perfil do solo (Cuenca \& Lovera, 2010). Os dados encontrados na área de estudo estão de acordo com estas hipóteses, já que foi registrada diminuição da densidade de FMA com o aumento da profundidade, sendo a densidade de FMA correlacionada com a quantidade de raízes finas.

As características morfológicas das raízes finas são influenciadas pela fertilidade do solo (Eissenstat et al., 2000; Powers et al., 2005) e, portanto, pela colonização por FMA (Zangaro et al., 2005, 2008). As plantas que crescem em solos de baixa fertilidade, especialmente nas fases iniciais da sucessão vegetal, geralmente apresentam alta alocação de biomassa nas raízes finas (Tilman, 1994; Comas \& Eissenstat, 2004). Essas informações ajudam a elucidar a relação positiva entre a biomassa de raízes finas e a densidade de esporos de FMA.

A variação sazonal da densidade de FMA está geralmente relacionada com a umidade de cada solo, com as características da planta hospedeira, com a combinação da intensidade e a duração do comprimento do dia, com as espécies de FMA e, ainda, com a tendência genética para umas espécies esporularem mais do que outras (Entry et al., 2002; Oehl et al., 2009; M. Moreira et al., 2009). No entanto, a influência da época do ano sobre a ocorrência de esporos de FMA ainda não é clara, havendo registros contraditórios. Alguns estudos citam que o maior número de esporos é observado na estação seca (C. F. Silva et al., 2006; Bonfim et al., 2010), enquanto outros relatam maior ocorrência na estação chuvosa (Trufem \& Viriato, 1990; Aidar et al., 2004; M. Moreira et al., 2006).

Na região amazônica, R. S. Maia et al. (2015) relataram a diminuição do número de esporos no período menos chuvoso, resultado similar ao encontrado na área deste estudo. Para Caproni et al. (2003), na estação chuvosa, é esperada menor densidade de esporos e maior abundância de outras estruturas (hifas), uma vez que estes são estruturas de resistência, desnecessárias neste período.

Santos \& Carrenho (2011) verificaram que as propriedades químicas do solo influenciam a estruturação das comunidades dos FMA. Estes autores e G. A. Silva et al. (2009) observaram que o teor de fósforo foi negativamente relacionado com o número de esporos. A baixa disponibilidade de $\mathrm{P}$ no solo é considerada um fator limitante para o crescimento das plantas em ecossistemas tropicais (Vitousek, 1984; Brundrett, 2002), sendo necessárias altas taxas de absorção deste elemento para suportar o crescimento 
rápido das espécies tropicais nas primeiras fases da sucessão (Ansanelo, 2010). No entanto, na área de estudo não houve variação da quantidade de $\mathrm{P}$ ao longo do processo sucessional, sendo que este nutriente pode não ser fator limitante para o crescimento da vegetação secundária neste trabalho.

O potássio, macronutriente participante do processo fotossintético, é citado por Malavolta et al. (1967) como estimulador da proliferação de FMA. Isto está de acordo com o que foi registrado na área de estudo, onde se observou uma relação positiva entre o potássio e a densidade de FMA.

Sieverding (1991) afirma que as diferenças observadas em savanas naturais na Colômbia podem ser parcialmente explicadas pelas diferenças na textura do solo. Segundo o autor, há uma tendência de menor densidade de esporos de FMA em solos arenosos. Os resultados obtidos na área de estudo corroboram essa informação, uma vez que a densidade de esporos está relacionada positivamente com o total de argila e negativamente com a quantidade de areia fina no solo. Dados similares também foram observados por Correia et al. (2004), em vegetações de cerrado, onde foram amostradas áreas muito e pouco argilosas.

\section{CONSIDERAÇÕES FINAIS}

Embora os fungos micorrízicos venham sendo usados como bioindicadores, os dados analisados demostram que o uso da densidade de FMA como indicador em florestas secundárias de terra firme, na região amostrada, limita-se à separação entre florestas secundária e primária, não sendo possível separar as diferentes etapas do processo de sucessão.

Observa-se que o período de coleta é determinante na densidade de FMA, havendo grande importância no fato de esta variável ser considerada nas interpretações dos dados.

A profundidade em que as amostras de solo para extração dos FMA são coletadas (0-10 e 10-20cm) é uma variável com forte influência na densidade de esporos. No entanto, o comportamento das amostras das duas profundidades é similar em relação à idade da floresta secundária, à etapa do processo sucessional e ao período de coleta.

\section{AGRADECIMENTOS}

Os autores agradecem ao Museu Paraense Emílio Goeldi; ao Programa de Pesquisas Ecológicas de Longa Duração (PELD) Caxiunã, pelo financiamento das coletas; ao Programa de Pós-Graduação em Ciências Ambientais, da Universidade Federal do Pará/Museu Paraense Emílio Goeldi/Empresa Brasileira de Pesquisa Agropecuária (UFPA) MPEG/EMBRAPA); e à Coordenação de Aperfeiçoamento de Pessoal de Nível Superior (CAPES), pela bolsa de doutorado à primeira autora.

\section{REFERÊNCIAS}

AIDAR, M. P. M., R. CARRENHO \& C. A. JOLY, 2004. Aspects of arbuscular mycorrhizal fungi in an Atlantic Forest chronosequence in Parque Estadual Turístico do Alto Ribeira (PETAR), SP. Biota Neotropica 4(2): 1-15. DOI: <http://dx.doi.org/10.1590/S1676$06032004000200005>$.

ANSANELO, A. P., 2010. Influência de fungos micorrízicos arbusculares de diferentes estádios da sucessão no crescimento e na absorção de nutrientes por Heliocarpus americanus L. (Tiliaceae): 1-58. Dissertação (Mestrado em Ciências Biológicas) Universidade Estadual de Londrina, Londrina.

AUGÉ, R., 2001. Water relations, drought and vesicular-arbuscular mycorrhizal symbiosis. Mycorrhiza 11(1): 3-42. DOI: <https://doi. org/10.1007/s005720100097>.

BAI, C., X. HE, H. TANG, B. SHAN \& L. ZHAO, 2009. Spatial distribution of arbuscular mycorrhizal fungi, glomalin and soil enzymes under the canopy of Astragalus adsurgens Pall. in the Mu Us sandland, China. Soil Biology \& Biochemistry 41(5): 941-947. DOI: <https:// doi.org/10.1016/j.soilbio.2009.02.010>.

BARBOSA, R. I. \&J. R. S. SANTOS, 2009. Biomassa de raízes em ecossistemas de savana - Protocolo (Versão 2): 1-15. Instituto Nacional de Pesquisas da Amazônia (Programa de Pesquisa em Biodiversidade), Boa Vista.

BERBARA, R., F. A. SOUZA \& H. FONSECA, 2006. Fungos micorrízicos arbusculares: muito além da nutrição. In: M. S. FERNANDES (Ed.): Nutrição mineral de plantas: 53-85. Sociedade Brasileira de Ciência do Solo, Viçosa.

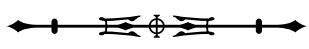


BONFIM, J. A., 2011. Diversidade de fungos micorrízicos arbusculares em áreas restauradas de Mata Atlântica, São Paulo, Brasil: 1-92. Dissertação (Mestrado em Solos e Nutrição de Plantas) - Universidade de São Paulo, Escola Superior de Agricultura Luiz de Queiroz, Piracicaba.

BONFIM, J. A., S. N. MATSUMOTO, J. M. LIMA, F. R. C. F. CÉSAR \&M. A. F. SANTOS, 2010. Fungos micorrízicos arbusculares (FMA) e aspectos fisiológicos em cafeeiros cultivados em sistema agroflorestal e a pleno sol. Bragantia 69(1): 201-206. DOI: <http://dx.doi. org/10.1590/S0006-87052010000100025>.

BRUNDRETT, M. C., 2002. Coevolution of roots and mycorrhizas of land plants. New Phytologist 154(2): 275-304. DOI: < https:// doi.org/10.1046/j.1469-8137.2002.00397.x>.

CAPRONI, A. L., A. A. FRANCO, R. L. L. BERBARA, S. B. TRUFEM, J. R. D. O. GRANHA \& A. B. MONTEIRO, 2003. Ocorrência de fungos micorrízicos arbusculares em áreas revegetadas após mineração de bauxita em Porto Trombetas, Pará. Pesquisa Agropecuária Brasileira 38(9): 1409-1418. DOI: <http://dx.doi. org/10.1590/S0100-204X2003001200007>.

CARDOSO, I. M., C. BODDINGTON, B. H. JANSSEN, O. OENEMA \& T. W. KUYPER, 2003. Distribution of mycorrhizal fungal spores in soils under agroforestry and monocultural coffee systems in Brazil. Agroforestry Systems 58(1): 33-43. DOI: <https://doi. org/10.1023/A:1025479017393>.

CARRENHO, R., S. M. GOMES-DA-COSTA, E. L. BALOTA \& A. COLOZZI-FILHO, 2010. Fungos micorrízicos arbusculares em agrossistemas brasileiros. In: J. O. SIQUEIRA, F. A. SOUZA, E. J. B. N. CARDOSO \& S. M. TSAI (Ed.): Micorrizas: 30 anos de pesquisas no Brasil: 153-214. Editora UFLA, Lavras.

CHAZDON, R., 2008. Chance and determinism in tropical forest succession. In: W. P. CARSON \& S. A. SCHNITZER (Ed.): Tropical forest community ecology: 384-408. Wiley-Blackwell Publishing, Oxford.

CHAZDON, R., 2012. Regeneração de florestas tropicais. Boletim do Museu Paraense Emílio Goeldi. Ciências Naturais 7(3): 195-218.

COIMBRA, J. L. M., V. Q. SOUZA, M. M. KOPP, J. G. C. SILVA, A. C. OLIVEIRA \& F. I. F. CARVALHO, 2006. Mean square expected values: an essential analysis. Ciência Rural 36(6): 1730-1738. DOI: <http://dx.doi.org/10.1590/S0103-84782006000600010>.

COMAS, L. H. \& D. M. EISSENSTAT, 2004. Linking fine root traits to maximum potential growth rate among 11 mature temperate tree species. Functional Ecology 18(3): 388-397. DOI: <https://doi.or $\mathrm{g} / 10.1111 / \mathrm{j} .0269-8463.2004 .00835 . x>$.

CORDEIRO, M. A. S., M. A. C. CARNeiro, H. B. PAULinO \& O. J. SAGGIN JUNIOR, 2005. Colonização e densidade de esporos de fungos micorrízicos em dois solos do cerrado sob diferentes sistemas de manejo. Pesquisa Agropecuária Tropical 35(3): 147-153.
CORREIA, M. A., A. REATTO, E. S. MARTINS, E. M. SILVA, L. S. CALDAS \& J. F. FAGG, 2004. Micorriza arbuscular: um bioindicador da fertilidade dos solos e da distribuição de árvores no Bioma Cerrado - Planaltina, DF. Boletim de Pesquisa e Desenvolvimento/ EMBRAPA Cerrados 132: 1-38.

COSTA, A., A. OLIVEIRA, M. COSTA, J. SILVA JUNIOR \& B. PORTELA, 2012. O clima da Floresta Nacional de Caxiuanã. In: INSTITUTO CHICO MENDES DE CONSERVAÇÃO DA BIODIVERSIDADE (ICMBIO). Plano de Manejo da Floresta Nacional de Caxiuanã: v. I - Diagnóstico: 108-114. ICMBIO, Brasília.

COSTA, M. L., D. C. KERN, H. BEHLING \& M. S. BORGES, 2002. Geologia. In: P. L. B. LISBOA (Ed.): Caxiuanã: populações tradicionais, meio físico e diversidade biológica: 179-206. Museu Paraense Emílio Goeldi, Belém.

CUENCA, G. \& M. LOVERA, 2010. Seasonal variation and distribution at different soil depths of arbuscular mycorrhizal fungi spores in a tropical sclerophyllous shrubland. Botany 88(1): 54-64. DOI: <https://doi.org/10.1139/B09-100>.

DANIELS-HETRICK, B. A. \&J. BLOOM, 1986. The influence of host plant on production and colonization ability of vesicular-arbuscular mycorrhizal spores. Mycologia 78(1): 32-36. DOI: <https://doi. org/10.2307/3793373>.

EISSENSTAT, D. M, C. E. WELLS, R. D. YANAI \&J. L. WHITBECK, 2000. Building roots in a changing environment: implications for root longevity. New Phytologist 147(1): 33-42. DOI: <https://doi.org/1 0.1046/j.1469-8137.2000.00686.x>.

EMPRESA BRASILEIRA DE PESQUISA AGROPECUÁRIA (EMBRAPA), 1997. Manual de métodos de análise de solo: 2. ed. Centro Nacional de Pesquisa de Solos/EMBRAPA, Rio de Janeiro.

ENTRY, J. A., P. T. RYGIEWIEZ, L. S. WATRUD \& P. K. DONNELLY, 2002. Influence of adverse soil conditions on the formation and function of arbuscular mycorrhizas. Advances in Environmental Research 7(1): 123-138. DOI: <https://doi.org/10.1016/S1093-0191(01)00109-5>.

FREITAS, R. O., 2005. Associações entre fungos micorrízicos arbusculares e espécies pioneiras em capoeiras na Amazônia Central: 1-80. Dissertação (Mestrado em Ciências de Florestas Tropicais) - Instituto Nacional de Pesquisas da Amazônia, Manaus.

GERDEMANN, J. \& T. NICOLSON, 1963. Spores of mycorrhizal Endogone species extracted from soil wet sieving and decanting. Transactions of Britsh Mycological Society 46(2): 235-244. DOI: $<$ https://doi.org/10.1016/S0007-1536(63)80079-0>.

GOTELLI, N. J. \& A. M. ELLISON, 2004. Princípios de estatística em Ecologia. Artmed Editora, Porto Alegre.

GUADARRAMA, P. \& F. J. ÁLVAREZ-SÁNCHES, 1999. Abundance of arbuscular mycorrhizal fungi spores in different environments in a tropical rain forest, Veracruz, Mexico. Mycorrhiza 8(5): 267-270. DOI: <https://doi.org/10.1007/s005720050244>.

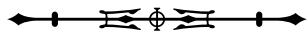


JANOS, D., 1980. Vesicular-arbuscular mycorrhizae affect lowland tropical rain forest plant growth. Ecology 61(1): 151-162. DOI: $<$ https://doi.org/10.2307/1937165>.

JANOS, D., 1996. Mycorrhizas, succession, and the rehabilitation of deforested lands in the humid tropics. In: J. FRANKLAND, N. MAGAN \& G. GADD (Ed.): Fungi and environmental change: 129-162. Cambridge University Press, for British Mycological Society, Cambridge.

JASPER, D. A., L. K. ABBOTT \& A. D. ROBSON, 1989. Soil disturbance reduces the infectivity of external hyphae of vesiculararbuscular mycorrhizal fungi. New Phytologist 112(1): 93-99. DOI: <https://doi.org/10.1111/j.1469-8137.1989.tb00313.x>.

JENKINS, W., 1964. A rapid centrifugation technique for separating nematodes from soil. Plant Disease Reporter 48(9): 1-692.

KENNARD, D., 2002. Secondary forest succession in a tropical dry forest: patterns of development across a 50-year chronosequence in lowland Bolivia. Journal of Tropical Ecology 18(1): 53-66. DOI: <https://doi.org/10.1017/S0266467402002031>.

KHURANA, E. \& J. S. SINGH, 2006. Impact of life-history traits on response of seedlings of five tree species of tropical dry forest to shade. Journal of Tropical Ecology 22(6): 653-661.

LANLY, J. P., 1982. Tropical forest resources. Food and Agriculture Organization (FAO Forestry Paper, 30), Rome.

LISBOA, P. L. B., 1997. A Estação Científica Ferreira Penna In: P. L. B. LISBOA (Org.): Caxiuanã: 23-49. Museu Paraense Emílio Goeldi, Belém.

MAIA, L. \& A. YANO-MELO, 2005. Role of arbuscular mycorrhizal fungi in saline soils. In: V. S. MEHROTRA (Ed.): Mycorrhizas: role and applications: 282-302. Allied Publishers, New Delhi.

MAIA, R. S., S. S. VASCONCELOS \& C. J. R. CARVALHO, 2015. Frações de fósforo e simbiose micorrízica em floresta secundária em resposta a disponibilidade de água e nutrientes na Amazônia Oriental. Acta Amazonica 45(3): 255-264. DOI: <http://dx.doi. org/10.1590/1809-4392201402894>

MALAVOLTA, E., H. P. HAAG, F. A. F. MELLO \& M. O. C. BRASIL SOBRINHO, 1967. Nutrição mineral de algumas culturas tropicais. Ed. Pioneira, São Paulo.

MEDEIROS, P. S., 2016. Indicadores ambientais para funcionalidade ecológica em florestas secundárias de diferentes idades na Amazônia Oriental: 1-247. Tese (Doutorado em Ciências Ambientais) - Universidade Federal do Pará/Museu Paraense Emílio Goeldi/Empresa Brasileira de Pesquisa Agropecuária, Belém.

MILLER, R. M. \& M. KLING, 2000. The importance of integration and scale in the arbuscular mycorrhizal symbiosis. Plant and Soil 226(2): 295-309. DOI: <https://doi. org/10.1023/A:1026554608366>.
MIRANDA, J. C. C., 2008. Cerrado: micorriza arbuscular, ocorrência e manejo: 1-169. EMBRAPA Cerrados, Planaltina.

MIRANDA, J. C. C., L. VILELA \& L. N. MIRANDA, 2005. Dinâmica e contribuição da micorriza arbuscular em sistemas de produção com rotação de culturas. Pesquisa Agropecuária Brasileira 40(10): 1005-1014. DOI: <http://dx.doi.org/10.1590/S0100$204 \times 2005001000009>$

MORAES, B., R. M. SILVA, J. B. RIBEIRO \& M. L. RUIVO, 2009. Variabilidade de precipitação na Floresta de Caxiuanã. In: P. L. LISBOA (Org.): Caxiuanã: desafios para a conservação de uma floresta nacional na Amazônia: 91-97. MPEG, Belém.

MOREIRA, F. \& J. SIQUEIRA, 2006. Microbiologia e bioquímica do solo: 1-729. UFLA, Lavras.

MOREIRA, M., D. BARETTA, S. M. TSAI \& E. J. B. N. CARDOSO, 2006. Spore density and root colonization by arbuscular mycorrhizal fungi in preserved or disturbed Araucaria angustifolia (Bert.) $\bigcirc$. Ktze. ecosystems. Scientia Agricola 63(4): 380-385. DOI: < http://dx.doi. org/10.1590/S0103-90162006000400009>

MOREIRA, M., D. BARETTA, S. M. TSAI \& E. J. B. N. CARDOSO, 2009. Arbuscular mycorrhizal fungal communities in native and in replanted Araucaria forest. Scientia Agricola 66(5): 677-684. DOI: <http://dx.doi.org/10.1590/S0103-90162009000500013>.

MUNYANZIZA, E., H. K. KEHRI \& D. J. BAGYARAJ, 1997. Agricultural intensification, soil biodiversity and agro-ecosystem function in the tropics: the role of mycorrhiza in crops and trees. Applied Soil Ecology 6(1): 77-85. DOI: <https://doi.org/10.1016/ S0929-1393(96)00152-7>.

MUTHUKUMAR, T., L. SHA, X. YANG, M. CAO, J. TANG \& Z. ZHENG, 2003. Distribution of roots and arbuscular mycorrhizal associations in tropical forest types of Xishuangbanna, southwest China. Apllied Soil Ecology 22(3): 241-253. DOI: <https://doi. org/10.1016/S0929-1393(02)00156-7>.

OEHL, F., E. SIEVERDING, K. INEICHEN, P. MADER, T. BOLLER \& A. WIEMKEN, 2003. Impact of land use intensity on the species diversity of arbuscular mycorrhizal fungi in agroecosystems of Central Europe. Applied and Environmental Microbiology 69(5): 2816-2824. DOI: <https://doi.org/10.1128/AEM.69.5.2816$2824.2003>$

OEHL, F., E. SIEVERDING, K. INECHEIN, T. BOLLER \& A. WIEMKEN, 2004. Impact of land use intensity on the species diversity of arbuscular mycorrhizal fungi in agroecosystems of Central Europe. Applied and Environmental Microbiology 69(5): 2816-2824. DOI: $<$ https://doi.org/10.1128/AEM.69.5.2816-2824.2003>.

OEHL, F., E. SIEVERDING, K. INEICHEN, E. A. RIS, T. BOLLER \& A. WIEMKEN, 2005. Community structure of arbuscular mycorrhizal fungi at different soil depths in extensively and intensively managed agroecosystems. The New Phytologist 165(1): 273-283. DOI: $<$ https://doi.org/10.1111/j.1469-8137.2004.01235.x>.

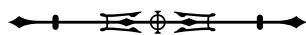


OEHL, F., E. SIEVERDING, K. INEICHEN, P. MADER, A. WIEMKEN \& T. BOLLER, 2009. Distinct sporulation dynamics of arbuscular mycorrhizal fungal communities from different agroecosystems in long-term microcosms. Agriculture, Ecosystems \& Environment 134(3-4): 257-268. DOI: <https:// doi.org/10.1016/j.agee.2009.07.008>.

PIOTROWSKI, J. S., Y. LEKBERG, M. J. HERNER, P. W. RAMSEY \& RILLIG, M. C. 2008. Dynamics of mycorrhizae during development of riparian forests along an unregulated river. Ecography 31(2): $245-$ 253. DOI: <https://doi.org/10.1111/j.0906-7590.2008.5262.x>.

POWERS, J. S., K. K. TRESEDER \& M. T. LERDAU, 2005. Fine roots, arbuscular mycorrhizal hyphae and soil nutrients in four neotropical rain forests: patterns across large geographic distance. New Phytologist 165(3): 913-921. DOI: <https://doi.org/10.1111 j.1469-8137.2004.01279.x>.

PURIN, S. \& M. C. RILLIG, 2007. The arbuscular mycorrhizal fungal protein glomalin: limitations, progress and a new hypothesis for its function. Pedobiologia 51(2): 123-130. DOI: < https://doi. org/10.1016/j.pedobi.2007.03.002>.

RILLIG, M. C. \& C. B. FIELD, 2003. Arbuscular mycorrhizae respond to plants exposed to elevated atmospheric $\mathrm{CO}_{2}$ as a function of soil depth. Plant Soil 254(2): 383-391. DOI: <https:// doi.org/10.1023/A:1025539100767>.

RILLIG, M. C., N. F. MARDATIN, E. F. LEIFHEIT \& P. M. ANTUNES, 2010. Mycelium of arbuscular mycorrhizal fungi increases soil water repellency and is sufficient to maintain water-stable soil aggregates. Soil Biology \& Biochemistry 42(7): 1189-1191. DOI: < https://doi. org/10.1016/j.soilbio.2010.03.027>.

SANTOS, F. E. F. \& R. CARRENHO, 2011. Diversidade de fungos micorrízicos arbusculares em remanescente florestal impactado (Parque Cinqüentenário - Maringá, Paraná, Brasil). Acta Botanica Brasilica 25(2): 508-516. DOI: <http://dx.doi.org/10.1590/S010233062011000200026>.

SIEVERDING, E., 1991. Vesicular-arbuscular mycorrhizae management in tropical agrosystems: 1-371. Eschborn, Federal Republic of Germany.

SILVA, C. F., M. G. PEREIRA, E. M. R. SILVA, M. E. F. CORREIA \& O. J. SAGGIN-JÚNIOR, 2006. Fungos micorrízicos arbusculares em áreas no entorno do Parque Estadual da Serra do Mar em Ubatuba (SP). Caatinga 19: 1-10.

SILVA, G. A., J. O. SIQUEIRA \& S. L. STÜRME, 2009. Eficiência de fungos micorrízicos arbusculares isolados de solos sob diferentes sistemas de uso na região do Alto Solimões na Amazônia. Acta Amazonica 39(3): 477-488. DOI: <http://dx.doi.org/10.1590/ S0044-59672009000300001>.

SMITH, S. E. \& D. READ, 2008. Mycorrhizal symbiosis: 3. ed.: 1-815. Academic Press, London.
SOUZA, F. A., I. SILVA \& R. BERBARA, 2008. Fungos micorrízicos arbusculares: muito mais diversos do que se imaginava. In: F. MOREIRA, J. SIQUEIRA \& L. BRUSSAARD (Ed.): Biodiversidade do solo em ecossistemas brasileiros: 483-536. UFLA, Lavras.

SOUZA, F. A., S. L. STÜRMER, R. CARRENHO \& S. F. TRUFEM, 2010. Classificação e taxonomia de fungos micorrízicos arbusculares e sua diversidade e ocorrência no Brasil. In: J. O. SIQUEIRA, F. A. SOUZA, E. J. CARDOSO \& S. M. TSAI (Ed.): Micorrizas: 30 anos de pesquisa no Brasil: 15-73. UFLA, Lavras.

STÜRMER, S. L., O. KLAUBERG FILHO, M. H. D. QUEIROZ \& M. M. D. MENDONÇA, 2006. Occurrence of arbuscular mycorrhizal fungi in soils of early stages of a secondary succession of Atlantic Forest in South Brazil. Acta Botanica Brasilica 20(3): 513-521. DOI: <http://dx.doi.org/10.1590/S0102$33062006000300002>$.

STÜRMER, S. L. \& J. O. SIQUEIRA, 2008. Diversidade de fungos micorrízicos arbusculares em ecossistemas brasileiros. In: F. M. S. MOREIRA, J. O. SIQUEIRA \& L. BRUSSARD (Ed.): Biodiversidade do solo em ecossistemas brasileiros: 537-584. Universidade Federal de Lavras, Lavras.

THE ANGIOSPERM PHYLOGENY GROUP (APG), 2009. An update of the Angiosperm Phylogeny Group classification for the orders and families of flowering plants: APG III. Botanical Journal of Linnean Society 161(2): 105-121. DOI: <https://doi.org/10.11 $11 / j .1095-8339.2009 .00996 . x>$.

TILMAN, D., 1994. Competition and biodiversity in spatially structured habitats. Ecology 75(1): 2-16. DOI: <https://doi. org/10.2307/1939377>

TRUFEM, S. F. B. \& A. VIRIATO, 1990. Fungos micorrízicos vesículo-arbusculares da Reserva Biológica do Alto da Serra de Paranapiacaba, São Paulo, Brasil. Revista Brasileira de Botânica 13: 49-54.

VITOUSEK, P. M., 1984. Litterfall, nutrient cycling, and nutrient limitation in tropical forests. Ecology 65(1): 285-298. DOI: $<$ https://doi.org/10.2307/1939481>.

WILDI, O., 2002. Modelling succession from pasture to forest in time and space. Community Ecology 3(2): 181-189.

YANG, F. Y., G. Z. LI, D. E. ZHANG, P. CHRISTIE, X. L. LI \& J. P. 2010. Geographical and plant genotype effects on the formation of arbuscular mycorrhiza in Avena sativa and Avena nuda at different soil depths. Biology and Fertility of Soils 46(5): 435-443. DOI: <https://doi.org/10.1007/s00374-010-0450-3>.

ZANGARO, W., S. M. A. NISIZAKI, J. C. B. DOMINGOS \& E. M. NAKANO, 2002. Micorriza arbuscular em espécies arbóreas nativas da bacia do rio Tibagi, Paraná. Cerne 8(1): 77-87.

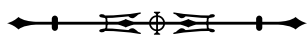


ZANGARO, W., F. R. NISHIDATE, F. R. S. CAMARGO, G. G. ROMAGNOLI \& J. VANDRESEN, 2005. Relationships among arbuscular mycorrhizas, root morphology and seedling growth of tropical native woody species in southern Brazil. Journal of Tropical Ecology 21(5): 529-540. DOI: <https://doi.org/10.1017/ S0266467405002555>.

ZANGARO, W., R. L. D. ASSIS, M. C. GONÇALVES, G. ANDRADE \& M. A. NOGUEIRA, 2008. Changes in arbuscular mycorrhizal associations and fine root traits in sites underdifferent plant successional phases in southern Brazil. Mycorrhiza 19(1): 37-45. DOI: <https://doi.org/10.1007/s00572-008-0202-5>.
ZANGARO, W., M. A. NOGUEIRA \& G. ANDRADE, 2009. Arbuscular mycorrhizal fungi as biofertilizers in revegetation programmes. In: M. K. RAI (Org.): Advances in fungal biotechnology: 1. ed.: 351-378. International Publishing House, New Delhi.

ZANGARO, W. \& M. MOREIRA, 2010. Micorrizas arbusculares nos biomas Floresta Atlântica e Floresta de Araucária. In: J. O. SIQUEIRA, F. A. SOUZA, E. J. B. N. CARDOSO \& S. M. TSAI (Ed.): Micorrizas: 30 anos de pesquisas no Brasil: 279-310. Editora UFLA, Lavras.

ZAR, J. H., 2009. Biostatistical analysis. Prentice-Hall, New Jersey. 
